\title{
Characteristic Relations for Nonperiodic Solutions of Mathieu's Equation*
}

\author{
T. Tamir and H. C. Wang \\ Electrophysics Department, Polytechnic Institute of Brooklyn, Brooklyn, N.Y.
}

(October 30, 1964)

\begin{abstract}
A simple compact formulation is presented for the eigenvalue $\lambda$ of the Mathieu differential equation $y^{\prime \prime}+\left(\lambda-2 h^{2} \cos 2 \theta\right) y=0$. The relations obtained thereby are employed to examine certain characteristic features of the solutions $y=e^{i \nu \theta} \sum_{n} c_{n} e^{2 i n \theta}$ in both stable ( $\nu$ real) and unstable ( $\nu$ complex $)$ regions. In the vicinity of the boundaries ( $\nu=m=$ integer) which separate these regions, the expressions derived for $\lambda$ are nonsingular and continuous; at the boundaries, these expressions are identical to available expansions which become therefore special cases of a more general formulation. The results are used to determine the magnitudes of the harmonic coefficients $c_{n}$ and the instability intervals. The latter are shown to be intimately related to restrictions on the complex value of the characteristic exponent $\nu$. In addition, simple approximations that are suitable for perturbation problems $\left(h^{2}\right.$ small $)$ are derived for all of the characteristic quantities.
\end{abstract}

\section{Introduction}

Hill's differential equation and its special case, the Mathieu equation with real coefficients

$$
\frac{d^{2} y}{d \theta^{2}}+\left(\lambda-2 h^{2} \cos 2 \theta\right) y=0
$$

were first examined in connection with the vibration of elliptic membranes $[1]^{1}$ and the motion of celestial bodies [2]. More recent studies were motivated by applications in the fields of acoustical and electromagnetic diffraction, frequency modulation, electromechanical transducer design, elliptic waveguides, etc.; since it is impractical to provide a complete bibliography within the present framework, the reader is referred to several excellent textbooks [3-6], which contain lengthy lists of references.

Most of the investigations of the Mathieu equation (1.1) were concerned primarily with only a very limited range of solutions - the periodic ones. Thus, if the solution to (1.1) is expressed in the Floquet form

$$
y(\theta)=e^{ \pm i \nu \theta} \sum_{n=-\infty}^{\infty} c_{n}( \pm i \nu) e^{2 i n \theta}
$$

where the \pm signs relate to the two possible characteristic solutions, these will be periodic whenever the characteristic exponent $\nu$ is an integer $(\nu=m=0,1,2,3, \ldots)$. In those restricted cases, the solutions $y(\theta)$ were extensively tabulated [7].

Of a somewhat different type are problems which do not require periodic solutions; in those cases, $\nu$ is not necessarily a real integer and it may also become complex. Thus, the Mathieu equation (1.1) may express the propagation of electromagnetic waves in a medium that possesses dielectric or magnetic properties which vary periodically $[8,9,10]$; in that case, $\theta$ represents a

*This paper is based on a portion of a dissertation submitted by H. C. Wang in partial fulfillment of the requirements for the Ph. D. (Electrophysics) degree at the Polytechnic Institute of Brooklyn, New York.

${ }^{1}$ Figures in brackets indicate the literature references at the end of this paper. 
space variable and $c_{n}( \pm i \nu)$ refer to the amplitudes of the space harmonics. An analogous situation occurs in the case of certain types of oscillators [11] (particularly those involving parametric action) for which $\theta$ is interpreted as a time variable whereas $c_{n}( \pm i \nu)$ relate to the amplitudes of the temporal harmonics. In all of these cases, tabulated results are few and restricted in range $[6,12]$.

The significance of the characteristic exponent $\nu$ can usually be related directly to the wave character of the physical phenomena described by the Mathieu equation (1.1). Hence the determination of $\nu$, i.e., its relation in terms of $\lambda$ and $h^{2}$ or, alternatively, the determination of $\lambda=\lambda\left(\nu, h^{2}\right)$, are usually of considerable importance. It is also recognized that, when a forcing term is present, the source (generator) element usually prescribes the wavelike nature of the phenomena as reflected through $\nu$; hence $\lambda$, rather than $\nu$, represents the unknown physical quantity to be determined. One may then employ expressions that are found in the literature [1-7] and which relate $\lambda$ in terms of power series of $h^{2}$ for integral values of $\nu$. For $\nu$ not an integer, the power series

$$
\lambda=\nu^{2}+\frac{1}{2\left(\nu^{2}-1\right)} h^{4}+\frac{5 \nu^{2}+7}{32\left(\nu^{2}-1\right)^{3}\left(\nu^{2}-4\right)} h^{8}+\ldots .
$$

is available; however, this relation converges poorly for $\nu$ close to an integer and its applicability is therefore limited.

The present paper presents a method for deriving explicit expressions for $\lambda=\lambda\left(\nu, h^{2}\right)$ which hold for arbitrary values of $\nu$. The expansions involved are based on the continued fraction relations [3-7] of the Mathieu equation and is an extension of a method for obtaining power series for $\nu$ integer [13]. The expressions obtained herein are not singular for $\nu$ integer, they are applicable for both real and complex values of $\nu$ and appear to converge rapidly. A different method which also converges rapidly was recently proposed by Schäfke, Ebert, and Groh [14]; in contrast to their results, the expressions presented here are explicit and they are particularly simple when approximate expressions are required for small $h^{2}$.

In addition to expansions for the eigenvalue $\lambda$, other characteristic parameters can be found via the expansions given here. Thus, the extent of the range for complex values of $\nu$ is discussed, as well as the relation between all of the harmonic amplitudes $c_{n}( \pm i \nu)$. It is also shown that, in the limit of $\nu$ becoming an integer, the results presented here are identical to those already available [1-7] for the Mathieu functions and it is suggested that the present method leads to a more systematic approach for future computations of the pertinent series.

Certain physical interpretations, notation and the representation of the various possible solutions are discussed in connection with the so-called "stability chart" in section 2. The derivation of the expansions for $\lambda=\lambda\left(\nu, h^{2}\right)$ is presented in section 3 and the pertinent formulas for small $h^{2}$ are given in section 4. Simplified expansions for $\nu$ integer are discussed in section 5 and the range for $\nu$ complex is evaluated in section 6 . The magnitudes of the harmonics $c_{n}( \pm i \nu)$ are dealt with in section 7. Finally, some comments are made in section 8 concerning the range for $h^{2}$ wherein the relations presented here are expected to be valid and to converge rapidly.

\section{Stability Chart}

The harmonic amplitudes $c_{n}=c_{n}( \pm i \nu)$ are interrelated via the infinite continued fraction expansions [3-6]:

$$
\begin{aligned}
& \frac{c_{n}}{c_{n-1}}=-\frac{h^{2}}{L_{n}}-\frac{h^{4}}{\mid L_{n+1}}-\frac{h^{4}}{L_{n+2}} \ldots . . \\
& \frac{c_{n}}{c_{n+1}}=-\frac{h^{2}}{L_{n}}-\frac{\left.h^{4}\right\rfloor}{L_{n-1}}-\frac{h^{4}}{L_{n-2}} \ldots \ldots
\end{aligned}
$$

where

$$
L_{n}=(\nu+2 n)^{2}-\lambda
$$


By combining eqs (2.1) and (2.2), one obtains

$$
\frac{L_{n}}{h^{4}}=\frac{1}{L_{n+1}}-\frac{h^{4} \mid}{\mid L_{n+2}} \ldots \ldots+\frac{1}{L_{n-1}}-\frac{h^{4} \mid}{\mid L_{n-2}} \ldots \ldots
$$

which is essentially an equation for $\lambda=\lambda\left(\nu, h^{2}\right)$. By solving it, one may obtain the eigenvalue $\lambda$ and thence the harmonic amplitudes $c_{n}$ via (2.1) or (2.2).

Relation (2.4) is often presented [3-9] in the form of a so-called "stability chart", as shown in figure 1. This diagram illustrates some of the properties associated with the eigenvalue $\lambda$ and the characteristic exponent $\nu$. Thus, for $h^{2}$ and $\lambda$ real, $\nu$ may be either real or complex. In the latter case:

$$
\nu=m+i \alpha
$$

where $\alpha$ is real and $m=0,1,2,3 \ldots$. For simplicity, the real part of $\nu$ will always be taken as positive, in both the real and the complex $\nu$ cases.

Regions with real and complex values of $\nu$ are called "stable" and "unstable" regions (or bands), respectively, in the literature [3-12]. A systematic definition of this terminology can be traced to van der Pol and Strutt [15]; the justification is afforded by the fact that, if $\nu$ is complex, the characteristic solutions in eq (1.2) are not bounded as $|\theta| \rightarrow \infty$. It is recognized that the "unstable" solutions should not necessarily be neglected in actual physical situations. Thus, when $\theta$ is bounded, both complex values of $\pm \nu$ are admissible; also, in certain unbounded situations, one of the solutions may be retained and only the other need be rejected. The latter case is illustrated by the radiation condition in electromagnetic fields [10].

From figure 1, one sees that unstable regions (bands) alternate with stable ones. The lines $\nu=m$ consist of two separate branches (for all $m \neq 0$ ) which join on the $\lambda$ axis and contain a region with $\nu$ complex $(=m+i \alpha)$; it may be shown that the two $\nu=m$ lines touch each other on the $\lambda$-axis with an order of tangency of $m-1$. As a consequence, for a fixed $h^{2}$, the width of the unstable band decreases as $m$ increases.

As indicated in figure 1 , the curves for $\nu=$ const. exhibit different variations in the stable and unstable regions. Those with $\nu$ real start from the $\lambda$-axis (at $\left.\nu^{2}=\lambda\right)$ and proceed towards increasing values of $h^{2}$; on the other hand, the curves for $\nu$ complex exhibit a minimum with respect to $h^{2}$ and are double-valued with respect to $\lambda$.

Whereas the stability diagram may be used directly $[9,10]$ to obtain one of the parameters $\lambda, \nu$, or $h^{2}$ when the other two are given, this procedure usually leads to a graphical construction and therefore its accuracy and convenience are somewhat limited. Instead, simple analytical expressions for $\lambda=\lambda\left(\nu, h^{2}\right)$ may be used and their derivation is given in the following section.

FIGURE 1. The Mathieu stability diagram.

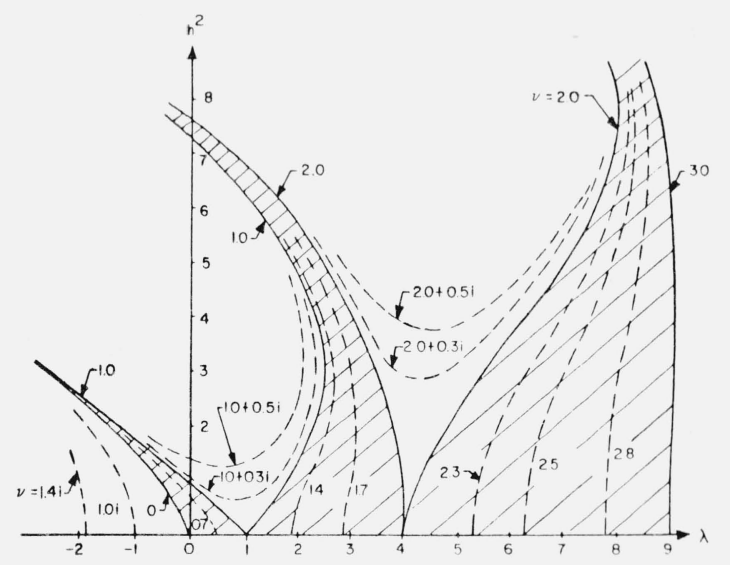




\section{Relations for the Eigenvalue $\lambda$}

In the present section, relations in the form $\lambda=\lambda\left(\nu, h^{2}\right)$ are obtained via the continued fractions of eq (2.4). When properly expanded as power series, these relations lead to equations for the eigenvalue $\lambda$ which, unlike eq (1.3), are not singular at the band edges $(\nu=m=0,1,2,3 \ldots$. . In order to better appreciate the procedure employed here, it is pertinent to illustrate first the derivation of eq (1.3).

By setting $n=0$ in eq (2.4), one obtains

$$
L_{0}=\nu^{2}-\lambda=\frac{h^{4}}{L_{1}}-\frac{h^{4}}{L_{2}}-\frac{h^{4}}{\mid L_{3}}-\cdots+\frac{h^{4} \mid}{L_{-1}}-\frac{\left.h^{4}\right\rfloor}{L_{-2}}-\frac{h^{4}}{\mid L_{-3}} \ldots .
$$

which may be written as

$$
\lambda=\nu^{2}-h^{4}\left(K^{+}+K^{-}\right)
$$

where

$$
K^{ \pm}=\frac{1}{L_{ \pm 1}}-\frac{h^{4}}{L_{ \pm 2}}-\frac{h^{4}}{L_{ \pm 3}} \ldots . .
$$

Equation (3.2) is now viewed as an iteration formula

$$
\lambda_{i+1}=\nu^{2}-h^{4}\left[K^{+}\left(\lambda_{i}\right)+K^{-}\left(\lambda_{i}\right)\right]
$$

for finding $\lambda_{i+1}$ when a trial value $\lambda_{i}(i=0,1,2,3 \ldots$. ) is assumed. To obtain an analytical result in terms of a power series in $h^{2}$, it is necessary to expand $K^{ \pm}$as a power series. This is achieved via a useful series expansion for continued fractions [16] which, in the present case, is written as

$$
\begin{aligned}
K^{ \pm}=\frac{1}{L_{ \pm 1}}+\frac{h^{4}}{L_{ \pm 1}^{2}\left(L_{ \pm 2}-\frac{h^{4}}{L_{ \pm 1}}\right)}+\frac{h^{8}}{L_{ \pm 1}^{2}\left(L_{ \pm 2}-\frac{h^{4}}{L_{ \pm 1}}\right)^{2}\left(L_{ \pm 3}-\frac{h^{4}}{L_{ \pm 2}}-\frac{h^{4}}{L_{ \pm 1}}\right)} \\
+\frac{h^{12}}{L_{ \pm 1}^{2}\left(L_{ \pm 2}-\frac{h^{4}}{L_{ \pm 1}}\right)^{2}\left(L_{ \pm 3}-\frac{h^{4}}{L_{ \pm 2}}-\frac{h^{4}}{L_{ \pm 1}}\right)^{2}\left(L_{ \pm 4}-\frac{h^{4}}{L_{ \pm 3}}-\frac{h^{4}}{L_{ \pm 2}}-\frac{h^{4}}{L_{ \pm 1}}\right)}+\ldots
\end{aligned}
$$

This expansion may be cast in the more compact form

$$
K^{ \pm}=\sum_{k=0}^{\infty} B_{ \pm k} B_{ \pm(k+1)} h^{4 k}
$$

where

$$
B_{0}=1 ; B_{ \pm k}=\prod_{j=1}^{k} A_{ \pm j}
$$

and

$$
\begin{gathered}
A_{ \pm j}=\frac{1}{L_{ \pm j}}-\frac{h^{4}}{L_{ \pm(j-1)}}-\frac{h^{4}}{L_{ \pm(j-2)}} \ldots . .-\frac{h^{4}}{L_{ \pm 2}}-\frac{h^{4}}{L_{ \pm 1}} \\
(j=1,2,3 \ldots ; k=0,1,2, \ldots)
\end{gathered}
$$

and it is understood that $A_{ \pm 1}=\frac{1}{L_{ \pm 1}}$.

It is recognized that the expansion in eqs (3.5) or (3.6) would be a power series in terms of $h^{4}$ if terms with $h^{4}$ did not also appear in $A_{ \pm j}$. However, if $h^{4}$ is sufficiently small, one may employ the binomial serres expansion to convert the above in a power series. Thus: 


$$
\begin{array}{r}
K^{+}=\frac{1}{L_{1}}+\frac{h^{4}}{L_{1}^{2} L_{2}}\left(1-\frac{h^{4}}{L_{1} L_{2}}\right)^{-1}+\frac{h^{8}}{L_{1}^{2} L_{2}^{2} L_{3}}\left(1-\frac{h^{4}}{L_{1} L_{2}}\right)^{-2}\left[1-\frac{h^{4}}{L_{2} L_{3}}\left(1-\frac{h^{4}}{L_{1} L_{2}}\right)^{-1}\right]^{-1}+\cdots \\
=\frac{1}{L_{1}}+\frac{h^{4}}{L_{1}^{2} L_{2}}+\left(1+\frac{L_{3}}{L_{1}}\right) \frac{h^{8}}{L_{1}^{2} L_{2}^{2} L_{3}}+\left(1+\frac{L_{4}}{L_{2}}+2 \frac{L_{3} L_{4}}{L_{1} L_{2}}+\frac{L_{3}^{2} L_{4}}{L_{1}^{2} L_{2}}\right) \frac{h^{12}}{L_{1}^{2} L_{2}^{2} L_{3}^{2} L_{4}}+0\left(h^{16}\right)
\end{array}
$$

and a similar power series for $K^{-}$. Introducing these series into eq (3.4), one obtains the required iteration formula which yields $\lambda$ in terms of $\nu$ and a power series in $h^{4}$. The proper starting value for the iteration process is obviously $\lambda_{0}=\nu^{2}$ for the stable regions and $\lambda_{0}=m^{2}(m=0,1,2 \ldots$. for the unstable regions, since these are the values of $\lambda$ at $h=0$ (see fig. 1). It is observed that by taking these starting values of $\lambda_{0}$, the iteration formula (3.4) is in a form such that $\lambda_{i}$ is obtained to $0\left(h^{4 i}\right)$. Hence, if $\lambda$ is to be found to $0\left[h^{4(i+1)}\right]$, it is necessary to find the series expansions for $K^{ \pm}$only up to $0\left(h^{4 i}\right)$.

By using these considerations, one may verify that eq (3.2) yields formula (1.3). As already noted, however, this formula yields good results only for values of $\nu$ which are not too close to a nonzero integer. When this condition is satisfied and if $h^{2}$ is not too large, both the iteration (3.4) and eq (1.3) converge rapidly. This fast convergence is due to the fact that $\lambda_{i+1}$ in eq (3.4) was isolated out from the term $L_{0}$, as indicated in eq (3.1) and, of all $L_{n}$ terms, $L_{0}$ varies most rapidly with both $h^{2}$ and $\nu$, provided $0<$ real part of $\nu<1$. This property is verified by noting that, for small $h^{2}$, one may write $\lambda \cong \nu^{2}$ to yield

$$
L_{n} \cong(\nu+2 n)^{2}-\nu^{2}=4 n(\nu+n)
$$

which is most sensitive for $n=0$ and $n=-\nu=-m$, where $m$ is the integer that is closest to the real part of $\nu$. Hence, as long as $\nu$ is real and sufficiently smaller than unity, $L_{0}$ is the only sensitive term. However, in general both $L_{0}$ and $L_{-m}$ may become critical; hence, an iteration formulation such as eq (3.4) would have to be based on a value for $\lambda_{i+1}$ which is isolated out from both $L_{0}$ and $L_{-m}$. This is accomplished herein by employing certain properties of the pertinent functions $L_{n}$, as discussed below.

Consider first the problem of isolating the term $L_{0}$ out of a continued fraction such as eqs (2.1) or (2.2). If $|n|$ is large, $L_{0}$ will appear far down in the expansion and the process of finding its value will become quite tedious. It is therefore convenient to extend the concept of $\mathrm{K}^{+}$of eq (3.3) and define

$$
K_{n}=\frac{1\rfloor}{L_{n}}-\frac{\left.h^{4}\right\rfloor}{\mid L_{n+1}}-\frac{\left.h^{4}\right\rfloor}{\mid L_{n+2}}-\cdots
$$

where the subscripts of the denominators form an increasing sequence. The term $L_{0}$ will appear only if $n \leqslant 0$; one may then write (see appendix):

$$
K_{n}=A_{n}+\frac{B_{n}^{2} h^{-4 n}}{S-C_{n} h^{4}} \quad(n \leqslant 0)
$$

where the terms $A_{n}$ and $B_{n}$ are given in eqs (3.8) and (3.7), respectively, with

$$
\begin{gathered}
S=L_{0}-K_{1} h^{4} \\
A_{0}=C_{0}=0 ; C_{n}=\sum_{k=0}^{-(n+1)} B_{-k} B_{-(k+1)} h^{4 k}(n<0)
\end{gathered}
$$

and it is noted that the terms $A_{n}=A_{n}(\nu), B_{n}=B_{n}(\nu)$ and $C_{n}=C_{n}(\nu)$ were defined in such a manner that, if expanded as power series in $h^{4}$, their expansion will start with a free term. 
In addition to the simplification realized by writing $K_{n}$ in the form of eq (3.12), the terms $L_{n}$ possess a certain symmetry which is basic in the derivations obtained here. This feature is exhibited by taking $\nu$ as

$$
\nu=m+\Delta
$$

where

$$
\Delta={ }_{i \alpha(\text { for } \nu \text { complex }) .}
$$

For complex values of $\nu$, eqs (3.15) and (3.16) correspond to eq (2.5) and therefore $m$ denotes the pertinent unstable region. In the stable regions, $m$ is chosen so that $|\beta| \leqslant \frac{1}{2}$; hence $m$ is then the integer closest to $\nu$ in an arithmetic sense.

Defining the "complementary" value of $\nu$ as

$$
\bar{\nu}=m-\Delta=2 m-\nu,
$$

one recognizes that $\bar{\nu}$ corresponds to the complex conjugate value of $\nu$ if the latter is complex. Consider now any function $F=F(\nu)$; then

$$
\bar{F}=\bar{F}(\nu) \triangleq F(\bar{\nu})
$$

where $\triangleq$ indicates equality by definition. If $F(\nu)$ is a polynomial, one may separate it as

$$
F=F e+F o
$$

where $F e=F e(m, \Delta)$ and $F_{o}=F_{o}(m, \Delta)$ are the functions containing only even or odd powers of both $m$ and $\Delta$. For complex values of $\nu, F e$, and $F o$ refer to the real and imaginary parts of $F$, respectively. One then also has

$$
\begin{gathered}
\bar{F}=F e-F o \\
\tilde{F}^{2} \triangleq F \bar{F}=(F e)^{2}-(F o)^{2}
\end{gathered}
$$

and it is recognized that, if $\nu$ is complex, the modulus $\tilde{F}$ is identical to the absolute value $|F|$. Applying definitions (3.17) and (3.18) to $L_{n}$ in eq (2.3), one gets

$$
L_{n}=\overline{\mathrm{L}}_{-(m+n)}
$$

which is the "symmetry" feature alluded above. Its importance lies in the fact that, whereas only the first fraction (with increasing indices in $L_{n}$ ) of eq (2.4) is expressed via $K_{n}$ of eqs (3.11) and (3.12), the second (decreasing) continued fraction is expressible in terms of $\bar{K}_{n}$ via eq (3.22).

A crucial point in the above definitions is the fact that $\lambda$ is regarded as an independent parameter when taking the complementary of $F(\nu)$. In particular, one notes that $L_{n}=L_{n}(\nu, \lambda)$ which may also be written as $L_{n}=L_{n}[\nu, \lambda(\nu)]$; definition (3.18), however implies that $\bar{L}_{n}=L_{n}(\bar{\nu}, \lambda)=L_{n}[\bar{\nu}, \lambda(\nu)]$ and therefore $\bar{L}_{n} \neq L_{n}[\bar{\nu}, \lambda(\bar{\nu})]$.

The following discussion is more convenient if even and odd values of $m$ are viewed separately. 
(a) $m$ odd: $m=2 r-1(r=1,2,3 \ldots$. .

For odd values of $m$, eq (2.4) may be written, with $n=1-r$, as

$$
L_{1-r}-\frac{\left.h^{4}\right\rfloor}{L_{2-r}}-\frac{\left.h^{4}\right\rfloor}{\mid L_{3-r}}-\cdots \cdot=\frac{\left.h^{4}\right\rfloor}{L_{-r}}-\frac{\left.h^{4}\right\rfloor}{\left\lfloor L_{-1-r}\right.}-\frac{h^{4}}{\mid L_{-2-r}}-\cdots
$$

which, by using relations (3.11), (3.18), and (3.22) becomes

$$
\frac{1}{K_{1-r}}=\bar{K}_{1-r} h^{4}
$$

The sensitive $L_{-m}$ term is thus replaced with $\bar{L}_{0}$. One recalls that, for an iteration formula similar to that of eq (3.4), the term $\lambda_{i+1}$ needs to be isolated from the $L_{0}$ term, i.e., out of $S$ of eq (3.13) in the present case; in particular, since now $m \neq 0, \lambda_{i+1}$ will be obtained from the even part of $L_{0}$. Writing

$$
X=S-C_{1-r} h^{4}
$$

the required iterated eigenvalue is given, via eqs (2.3), (3.13), and (3.25), by

$$
\lambda^{(2 r-1)}=m^{2}+\Delta^{2}-X e-\left(K e_{1}+C e_{1-r}\right) h^{4}
$$

where $\lambda^{(m)}$ will henceforth be used to denote the pertinent eigenvalue appropriate to the $m$ th region, as defined in eqs (3.15) and (3.16). To find $X e$ in eq (3.26), it is realized that eq (3.24) leads, via eqs (3.12) and (3.25), to

$$
\left[A_{1-r}+\frac{B_{1-r}^{2} h^{4(r-1)}}{X}\right]\left[\bar{A}_{1-r}+\frac{\bar{B}_{1-r}^{2} h^{4(r-1)}}{\bar{X}}\right] h^{4}=1
$$

and the last relation is recognized to be a quadratic equation for $X e . \quad$ By solving it and substituting into eq (3.26), one finally obtains:

$$
\lambda_{ \pm}^{(2 r-1)}=(2 r-1)^{2}+\Delta^{2}-G^{\prime} e \cdot h^{4} \pm \sqrt{\left[\tilde{F}^{\prime} \cdot h^{2(2 r-1)}\right]^{2}+\left[2(2 r-1) \Delta-G^{\prime} o \cdot h^{4}\right]^{2}}
$$

where

$$
\begin{gathered}
F^{\prime}=\frac{B_{1-r}^{2}}{1-\tilde{A_{1-r}^{2}} h^{4}} \\
G^{\prime}=K_{1}+C_{1-r}-\overline{A_{1-r}} F^{\prime} h^{4(r-1)} .
\end{gathered}
$$

Result (3.28) is the required iteration formula for $m=2 r-1$; though more complicated, this result is analogous to the iteration in eq (3.4) for $m=0$. The \pm signs in $\lambda_{ \pm}^{(m)}$ refer to the two admissible eigenvalues for given $h^{2}$ and $\Delta$. As already discussed in conjunction with the stability chart, all $\lambda^{(m)}(m \neq 0)$ are double-valued for a given $\nu$ and $h^{2}$ in the unstable regions; the + and - signs then refer to the larger and the smaller values of $\lambda^{(m)}$, respectively. In the stable bands, $\lambda^{(m)}$ is single-valued and the two eigenvalues in eq (3.28) arise due to the choice of $m$ in eq (3.15); it is then easy to verify that $\lambda_{ \pm}^{(m)}$ correspond to $\beta \gtrless 0$, i.e., $\lambda_{+}^{(m)}$ applies if $m<\nu<m+\frac{1}{2}$, whereas $\lambda_{-}^{(m)}$ is pertinent if $m-\frac{1}{2}<\nu<m$. 
(b) $m$ even: $m=2 r(\mathrm{r}=1,2,3 \ldots)$

For even values of $m$, eq (2.4) is written, with $n=-r$ and relations (3.11), (3.18), and (3.22), as

$$
L_{-r}=\left(K_{1-r}+\bar{K}_{1-r}\right) h^{4}
$$

which, by employing the notation of eqs (3.12) and (3.26), becomes

$$
L_{-r}-2 A e_{1-r} h^{4}=\left(\frac{B_{1-r}^{2}}{X}+\frac{\bar{B}_{1-r}^{2}}{\bar{X}}\right) h^{4 r} .
$$

One then obtains again a quadratic with respect to $X e$ which yields a similar, but different, solution when compared with the $m$ odd case. By noting the value of $L_{-r}$, as defined via eq (2.3), one now obtains

where

$$
\lambda_{ \pm}^{(2 r)}=4 r^{2}+\Delta^{2}-G^{\prime \prime} e h^{4} \pm \sqrt{\left(\tilde{F}^{\prime \prime} h^{4 r}\right)^{2}+\left(4 r \Delta-G^{\prime \prime} o \cdot h^{4}\right)^{2}}
$$

$$
\begin{aligned}
F^{\prime \prime} & =\frac{B_{1-r}^{2}}{\Delta^{2}-\lambda_{ \pm}^{(2 r)}-2 A e_{1-r} h^{4}} \\
G^{\prime \prime} & =K_{1}+C_{1-r}+F^{\prime \prime} h^{4(r-1)} .
\end{aligned}
$$

Equation (3.32) is the required iteration formula for even values of $m$; the significance of the \pm signs in $\lambda_{ \pm}^{(m)}$ was already discussed in the paragraph following eq (3.29).

\section{Formulas for the Eigenvalue $\lambda^{(m)}$ for Small $h^{2}$}

Relations (3.28) and (3.32) are directly applicable for obtaining $\lambda^{(m)}$ to any desired accuracy by means of numerical methods. In addition, analytical formulations may be obtained for $\lambda^{(m)}$ provided $h^{2}$ is sufficiently small, this being particularly significant in certain physical situations. Thus, small $h^{2}$ occurs in problems wherein the periodicity with respect to the independent variable $\theta$ appears as a perturbation in an otherwise uniform configuration $\left(h^{2}=0\right)$; it is then clear (e.g., from the stability chart) that instability phenomena may appear in certain ranges even if the perturbation $h^{2}$ is exceedingly small.

To examine these effects, all of the functions appearing in eqs (3.28) and (3.32) are expanded as power series via relations which are analogous to eq (3.6) and (3.9). Using considerations similar to those described in the paragraph following eq (3.9), formulas (3.28) and (3.32) are then employed to yield relations for $\lambda^{(m)}$ up to any desired power of $h^{2}$. This method is applied in the present section to obtain expansion for $\lambda^{(m)}$ for small $h^{2}$ up to and including $\mathrm{O}\left(h^{4}\right)$. It is easy to verify that, if all terms of $\mathrm{O}\left(h^{6}\right)$ or smaller are neglected, it is sufficient to retain only first terms $\left(h^{0}\right)$ in the expansions for $K_{1}, A_{n}$, etc.; the approximations involved are commented upon in section 8 . The following results are thus obtained:

(a) In the stable regions ( $\nu$ real).

Formulas (3.4), (3.28) and (3.32) are used and expanded to $\mathrm{O}\left(h^{4}\right)$; the iterations need be employed only once and with a trial value of $\lambda_{0}{ }^{(m)}=\nu^{2}$. The exact definition of $\bar{L}_{n}$ is important in these derivations, as commented upon in the two paragraphs following eq (3.22).

$$
\begin{aligned}
& m=0: \quad 0 \leqslant \nu \leqslant \frac{1}{2} ; \quad \lambda^{(0)}=\nu^{2}-\frac{h^{4}}{2\left(1-\nu^{2}\right)}+\mathrm{O}\left(\frac{7}{128} h^{8}\right), \\
& m=1: \quad \frac{1}{2} \leqslant \nu_{-} \leqslant 1,1 \leqslant \nu+\leqslant \frac{3}{2} ; \\
& \lambda_{ \pm}^{(1)}=1+\left(1-\nu_{ \pm}\right)^{2}-\frac{\left(5-\nu_{ \pm}\right) h^{4}}{16\left(1+\nu_{ \pm}\right)\left(2-\nu_{ \pm}\right)} \\
& \pm \sqrt{h^{4}+\left[2+\frac{3 h^{4}}{16\left(1+\nu_{ \pm}\right)\left(2-\nu_{ \pm}\right)}\right]^{2}\left(1-\nu_{ \pm}\right)^{2} \pm O}\left(\frac{h^{6}}{64}\right),
\end{aligned}
$$


$m=2: \quad \frac{3}{2} \leqslant \nu_{-} \leqslant 2,2 \leqslant \nu_{+} \leqslant \frac{5}{2}$

$$
\begin{aligned}
\lambda_{ \pm}^{(2)}=4 & +\left(2-\nu_{ \pm}\right)^{2}+\frac{\left(7-\nu_{ \pm}^{2}\right) h^{4}}{\left(1-\nu_{ \pm}^{2}\right)\left(3-\nu_{ \pm}\right)} \\
& \pm \sqrt{\frac{h^{8}}{16\left(1-\nu_{ \pm}\right)^{2}}+\left[4+\frac{h^{4}}{6\left(1+\nu_{ \pm}\right)\left(3-\nu_{ \pm}\right)}\right]^{2}\left(2-\nu_{ \pm}\right)^{2}}-\mathrm{O}\left(\frac{379 \pm 384}{13824} h^{8}\right),
\end{aligned}
$$

$m \geqslant 3: \quad m-\frac{1}{2} \leqslant \nu_{-} \leqslant m, m \leqslant \nu_{+} \leqslant m+\frac{1}{2}$

$$
\lambda_{ \pm}^{(m)}=\nu_{ \pm}^{2}-\frac{h^{4}}{2\left(1-\nu_{ \pm}^{2}\right)}+\mathrm{O}\left[\frac{5 m^{2}+7}{32\left(m^{2}-1\right)^{3}\left(m^{2}-4\right)} h^{8}\right] \text {. }
$$

It is noted that, to $\mathrm{O}\left(h^{4}\right)$, only the results for $m=1$ and $m=2$ are different from eq (1.3). On the other hand, eqs (4.2) and (4.3) imply that eq (1.3) may lead to very erroneous results even for small perturbations; this relates to the regions which are close to $\nu=1$ and $\nu=2$, respectively.

The order of magnitude of the first neglected term in the above expansions is also indicated; the figures give the exact result for $\nu=m$ and it is expected that they yield a very good approximation for $\nu \neq m$.

(b) In the unstable regions $(\nu=m+i \alpha)$.

As illustrated in figure $1, h^{2}$ and $\nu$ may not be prescribed in a completely arbitrary fashion since, for $m \neq 0$ and a given $h^{2}, \alpha$ cannot exceed a certain value if $\lambda^{(m)}$ is to be real. The limiting $\alpha$ corresponds to the minimum of the constant $\nu$ curves in the unstable region; this value of $\alpha$ may be calculated by stipulating that the radicands in eqs (3.28) and (3.32) vanish, thus obtaining

$$
\alpha \leqslant w_{m} h^{2 m}+\mathrm{O}\left(h^{2 m+2}\right)
$$

where the equal sign refers to the limiting value and

$$
w_{m}=\frac{1}{2 m\left[2^{m-1}(m-1) !\right]^{2}}(\text { for all } m \neq 0) .
$$

Since the coefficients $w_{m}$ form a descending sequence, $\alpha$ is restricted to exceedingly small values for large $m$. This behavior is directly related to the extent of instability since $\alpha$ refers to the exponential increase of the solution (1.2); consequently, large values of $m$ (and therefore of $\lambda^{(m)}$ ) can accommodate only "weak" instabilities (at least for sufficiently small values of $h^{2}$ ).

With restriction (4.5) being accounted for, $\lambda^{(m)}$ may be obtained in the same fashion as in the stable regions, except that the trial values for the iteration are taken as $\lambda_{0}^{(0)}=-\alpha^{2}(m=0)$ and $\lambda_{0}^{(m)}$ $=m^{2}(m \neq 0)$. It is then obtained:

$$
\begin{array}{ll}
m=0: & \lambda^{(0)}=-\alpha^{2}-\frac{h^{4}}{2\left(1+\alpha^{2}\right)}+\mathrm{O}\left[\frac{\left(7-5 \alpha^{2}\right) h^{8}}{32\left(1+\alpha^{2}\right)^{3}\left(4+\alpha^{2}\right)}\right] \\
m=1: & \lambda_{ \pm}^{(1)}=1-\alpha^{2}-\frac{\left(8-\alpha^{2}\right) h^{4}}{\left(4+\alpha^{2}\right)\left(16+\alpha^{2}\right)} \pm \sqrt{h^{4}-(2 \alpha)^{2}\left[1+\frac{3 h^{4}}{\left(4+\alpha^{2}\right)\left(16+\alpha^{2}\right)}\right]^{2}} \mp \mathrm{O}\left(\frac{h^{6}}{64}\right) \\
m=2: & \lambda_{ \pm}^{(2)}=4-\alpha^{2}+\frac{2\left(12+\alpha^{2}\right) h^{4}}{\left(4+\alpha^{2}\right)\left(36+\alpha^{2}\right)} \pm \sqrt{\left(\frac{h^{4}}{4+\alpha^{2}}\right)^{2}-(4 \alpha)^{2}\left[1+\frac{2 h^{4}}{\left(4+\alpha^{2}\right)\left(36+\alpha^{2}\right)}\right]^{2}} \\
-0\left(\frac{379 \pm 384}{13824} h^{8}\right)
\end{array}
$$

$m \geqslant 3: \quad \lambda_{ \pm}^{(m)}=m^{2}+\frac{h^{4}}{2\left(m^{2}-1\right)}+\mathrm{O}\left[\frac{5 m^{2}+7}{32\left(m^{2}-1\right)^{3}\left(m^{2}-4\right)} h^{8}\right]$. 
Due to analogous considerations, the comments following eq (4.4) (with respect to $m \geqslant 3$ ) also apply here.

\section{Characteristic Curves for the Periodic Mathieu Functions $(\nu=m)$}

As a particular case, formulas (3.28) and (3.32) are applicable for obtaining the characteristic curves of the periodic Mathieu functions $(\nu=m)$. Observing that $\Delta \equiv 0$ in these cases, all of the odd functions $A \mathrm{o}_{n}, B \mathrm{o}_{n}$, etc., are identically zero and the formulas simplify considerably.

The notation for the band edges is generally given as $a_{m}=\left.\lambda_{+}^{(m)}(\nu)\right|_{\nu=m}$ and $b_{m}=\left.\lambda_{-}^{(m)}(\nu)\right|_{\nu=m}$ in the literature [3-6]. One then obtains

$$
\begin{aligned}
& a_{0}=-2 K_{1} h^{4}, \\
& b_{2 r-1}=(2 r-1)^{2}-\left(K_{1}+C_{1-r}\right) h^{4}-\frac{B_{1-r}^{2} h^{2(2 r-1)}}{1+A_{1-r} h^{2}}, \\
& a_{2 r-1}=(2 r-1)^{2}-\left(K_{1}+C_{1-r}\right) h^{4}+\frac{B_{1-r}^{2} h^{2(2 r-1)}}{1-A_{1-r} h^{2}}, \\
& b_{2 r}=4 r^{2}-\left(K_{1}+C_{1-r}\right) h^{4}, \\
& a_{2 r}= 4 r^{2}-\left(K_{1}+C_{1-r}\right) h^{4}+\frac{B_{1-r}^{2} h^{4 r}}{\frac{1}{2} a_{2 r}+A_{1-r} h^{4}}, \\
& \quad(r=1,2,3 \ldots) .
\end{aligned}
$$

These relations may be used to generate the Mathieu function expansions for the eigenvalues $a_{m}$ or $b_{m}$ in terms of a power series in $h^{2}$. It is then evident that certain known features are already apparent in formulas (5.1)-(5.5); thus, odd values of $m$ lead to expansions in terms of powers of $h^{2}$ whereas even values of $m$ lead to expansions in terms of powers of $h^{4}$. In fact, if these expansions are obtained iteratively with a first trial value of $\lambda_{0}=m^{2}$, the above formulas yield results which are identical with expressions available in the literature [3-7]. The identification follows upon recognizing that, for example, eqs (5.2)-(5.5) are a restatement of eqs (20-2, 21-24), respectively, on p. 723 of reference 6 .

The advantage of the present formulation is evident in that it represents a systematic technique for generating the power expansions for $\lambda^{(m)}$. Whereas it is recognized that all methods (including the present one) become quite involved and tedious if $a_{m}$ or $b_{m}$ need to be found in terms of large powers of $h^{2}$, it is nevertheless clear that formulas (5.1)-(5.5) are already expressed in such a concise and systematic manner that considerable simplification occurs.

\section{Instability Intervals}

The width of the band between each pair of the characteristic $\nu=m$ curves is of special importance since it yields information on the extent of the range of instability. For an arbitrary value of $h^{2}$, this instability interval is defined by

$$
W_{m}=a_{m}-b_{m}(m \neq 0)
$$

and is easily determined via relations (5.1) to (5.5) which yield:

$$
\begin{gathered}
W_{2 r-1}=\frac{2 B_{1-r}^{2}}{1-A_{1-r}^{2} h^{4}} h^{2(2 r-1)} \\
W_{2 r}=\frac{2 B_{1-r}^{2}}{a_{2 r}+2 A_{1-r} h^{4}} h^{4 r}
\end{gathered}
$$


Both results may be expressed also as

$$
W_{m}=2\left[\frac{h^{m}}{2^{m-1}(m-1) !}\right]^{2}+\mathrm{O}\left(h^{2 m+2}\right)
$$

which is obtained by taking the first term in a series expansion of eqs (6.2) or (6.3) in powers of $h^{2}$. It is observed that, to this order of approximation

$$
W_{m} \cong 4 m w_{m} h^{2 m}
$$

where $w_{m}$ are the coefficients in eq (4.6). Result (6.4) is identical to that already obtained by Bell [17], Levy and Keller [18] and Hochstadt [19] who used different approaches. In the present case, the instability interval $W_{m}$ turns out as a simple corollary to formulas (5.2)-(5.5) and the accuracy of eq (6.4) can be estimated as discussed in section 8 . It is also noted that $W_{m}$ is simply related to the maximum allowable value of $\alpha$ since, introducing eq (4.5) into eq (6.5), one has

$$
\alpha \leqslant \frac{W_{m}}{4 m}+\mathrm{O}\left(h^{2 m+2}\right) .
$$

Hence, as $m$ increases, the range of $\alpha$ becomes more restricted than that of $W_{m}$. Phrased differently, larger values of $\nu$ (or $\lambda$ ) lead to regions with decreasing unstable intervals; the extent of instability itself is proportional to the interval $W_{m}$, but inversely proportional to $\nu$ (or $\lambda$ ).

\section{Magnitude of Harmonics}

The magnitude of the harmonic coefficients $c_{n}( \pm i \nu)$ of eq (1.2) may be found by using the appropriate values of $\lambda^{(m)}$ obtained above. For simplicity, the $+\operatorname{sign}$ in $c_{n}( \pm i \nu)$ will be implied subsequently; hence, $c_{n}$ is taken to denote $c_{n}(i v)$. To find the coefficients $c_{n}(-i \nu)$, one may employ the identity $c_{n}(-i \nu)=c_{-n}(i \nu)$ which is proven in the literature [3-6].

Using eqs (2.1), (2.2), (3.11), and (3.22), one gets

$$
\begin{aligned}
\frac{c_{n}}{c_{n-1}} & =-h^{2} K_{n} \\
\frac{c_{n}}{c_{n+1}} & =\frac{\bar{c}_{-(m+n)}}{\bar{c}_{-(m+n+1)}}=-h^{2} \bar{K}_{-(m+n)}
\end{aligned}
$$

As clarified below, the $c_{o}$ and $c_{-m}$ harmonics turn out to be particularly important. For these harmonics, one obtains the following relations:

(a) For $m$ odd; $m=2 r-1(r=1,2,3 \ldots$. .).

By using eq (7.1) successively, one obtains

$$
\frac{c_{0}}{c_{1-r}}=\prod_{i=0}^{r-2} \frac{c_{-i}}{c_{-1-i}}=\left(-h^{2}\right)^{r-1} \prod_{i=0}^{r-2} K_{-i}
$$

and eq (7.2) similarly yields:

$$
\frac{c_{1-2 r}}{c_{1-r}}=\prod_{i=r}^{2 r-1} \frac{c_{-i}}{c_{1-i}}=\left(-h^{2}\right)^{r} \cdot \prod_{i=0}^{r-1} \bar{K}_{-i}
$$

so that, by division:

$$
R^{\prime}=\frac{c_{1-2 r}}{c_{0}}=\left\{\begin{array}{l}
-h^{2} \bar{K}_{0}(\text { for } r=1) \\
-h^{2} \bar{K}_{1-r} \prod_{i=0}^{r-2} \frac{\bar{K}_{-i}}{K_{-i}}(\text { for } r>1)
\end{array}\right.
$$


(b) For $m$ even; $m=2 r(r=1,2,3 \ldots)$.

In a manner similar to that for $m$ odd, one gets:

$$
\begin{aligned}
\frac{c_{0}}{c_{-r}} & =\prod_{i=0}^{r-1} \frac{c_{-i}}{c_{-1-i}}=\left(-h^{2}\right)^{r} \prod_{i=0}^{r-1} K_{-i} \\
\frac{c_{-2 r}}{c_{-r}} & =\prod_{i=r+1}^{2 r} \frac{c_{-i}}{c_{1-i}}=\left(-h^{2}\right)^{r} \prod_{i=0}^{r-1} \bar{K}_{-i}
\end{aligned}
$$

and, by division:

$$
R^{\prime \prime}=\frac{c_{-2 r}}{c_{0}}=\prod_{i=0}^{r-1} \frac{\bar{K}_{-i}}{K_{-i}}
$$

By using eqs (7.1), (7.2), (7.5), and (7.8), one may now express all $c_{n}$ by means of the following relations:

$$
\frac{c_{n}}{c_{0}}=\left\{\begin{array}{l}
\left(-h^{2}\right)^{n} \prod_{i=1}^{n} K_{i} \quad(n>0) \\
\left(-h^{2}\right)^{-n} \prod_{i=m+n}^{m-1} \bar{K}_{-i} \quad(-r<n<0) \\
\left(-h^{2}\right)^{m+n} R \prod_{i=-n}^{m-1} K_{-i} \quad(-m<n \leqslant-r) \\
\left(-h^{2}\right)^{-(m+n)} R \prod_{i=1}^{-(m+n)} \bar{K}_{i} \quad(n<-m)
\end{array}\right.
$$

and it is understood that $R$ stands for $R^{\prime}$ or $R^{\prime \prime}$ for odd or even values of $m$, respectively. The fundamental harmonic $c_{0}$ may be taken as unity or normalized via an orthogonal relationship [3-6]; all these normalization procedures, however, lead to a real value for $c_{0}$.

Equations (7.5) and (7.8) lead to the useful relation

$$
\tilde{R}^{2}=R \bar{R}=1
$$

which is obvious for $R=R^{\prime \prime}$; for $R=R^{\prime}$, this is verified by observing that eq (3.24) accounts for the extra $K_{1-r}$ term in eq (7.5). One therefore gets

$$
\tilde{c}_{n}^{2}=\tilde{c}_{-(m+n)}^{2}
$$

which is obtained via eq (7.9) and (7.10).

Result (7.11) is interesting since one recalls that, in the unstable bands, any function $F(\nu)$ leads to a complementary function $F(\bar{\nu})=\bar{F}(\nu)$ which is identical to $F^{*}(\nu)=F\left(\nu^{*}\right)$, where the asterisk denotes the complex conjugate. Consequently, the modulus $\tilde{F}(\nu)$ is then identical to the absolute value $|F(\nu)|$, so that

$$
\left|c_{n}\right|=\left|c_{-(m+n)}\right|
$$

in the unstable bands. The harmonic amplitudes are then equal in pairs, as shown in figure 2. Relation (7.12) holds up to and including the band edges $(\nu=m)$ where all variables are real; hence

$$
c_{n}= \pm c_{-(m+n)}
$$


since all terms are real and therefore any function $\bar{F}(\nu)=F^{*}(\nu)=F(\nu)$ at the band edges. The proper sign in eq (7.13) is determined by the sign of $R= \pm 1$ itself at the band edges. For $m$ odd, the sign of $R^{\prime}$ is given by the sign of $-h^{2} \bar{K}_{1-r}=-h^{2} K_{1-r}$ because the product term in eq (7.5) is obviously +1 at the band edge. By using eqs (3.12), (3.13), and (3.24), one has

$$
-h^{2} K_{1-r}=-A_{1-r} h^{2}-\frac{B_{1-r}^{2} h^{2(2 r-1)}}{(2 r-1)^{2}-\lambda_{ \pm}^{(2 r-1)}-\left(K_{1}+C_{1-r}\right) h^{4}}=R^{\prime}= \pm 1
$$

where $\lambda_{-}^{(2 r-1)}$ refers to $b_{2 r-1}$ and $\lambda_{+}^{(2 r-1)}$ refers to $a_{2 r-1}$ of eqs (5.2) and (5.3), respectively. It is easily seen by inspection that, in order to comply with eqs (5.2) and (5.3), $R^{\prime}=+1$ need be taken for $a_{2 r-1}$ whereas $R^{\prime}=-1$ is appropriate for $b_{2 r-1}$ in eq (7.14).

The even $m$ case is somewhat more complex. Checking for $K_{1-r}$, it is seen that $a_{2 r}$ of eq (5.5) leads to a finite value for $K_{1-r}$ but $b_{2 r}$ of eq (5.4) requires that $\left|K_{1-r}\right| \rightarrow \infty$. Hence $R^{\prime \prime}=+1$ for $a_{2 r}$ (since then all $K_{-i}=\bar{K}_{-i}$ are finite) but is yet undetermined for $b_{2 r}$. To clarify the latter situation, one uses eq (3.30) to find the limit

$$
\lim _{\Delta \rightarrow 0} \frac{\bar{K}_{1-r}}{K_{1-r}}=\lim _{\Delta \rightarrow 0}\left[\frac{L_{-r}}{h^{4} K_{1-r}}-1\right]=-1
$$

and since all $K_{-i}=\bar{K}_{-i}(i \neq r-1)$ in eq (7.8) at the edge pertinent to $b_{2 r}$, eq (7.15) leads to $R^{\prime \prime}=-1$ at that edge. In conclusion, one obtains that

$$
\begin{aligned}
& c_{n}=+c_{-(m+n)} \text { for } a_{m} \\
& c_{n}=-c_{-(m+n)} \text { for } b_{m}
\end{aligned}
$$

and therefore the function $y(\theta)$ of eq (1.2) is in the form of a Fourier cosine or sine expansion, respectively, on the right or left edge of the unstable regions.

A special feature of the $b_{2 r}$ edge is that, since $K_{1-r} \rightarrow \infty$, one gets from eq (7.6) that $c_{-r}=0$ which also agrees with the second of eqs (7.15); hence, the $c_{-r}$ harmonic vanishes at the left edge of all the unstable regions which are characterized by even values of $m=2 r$. It is appreciated that this harmonic is exceptional in that it is the only one which (for $m$ even) does not pair off with any other harmonic in the unstable regions but, as shown in figure 2 and eq (7.12), it can be said to pair with itself.

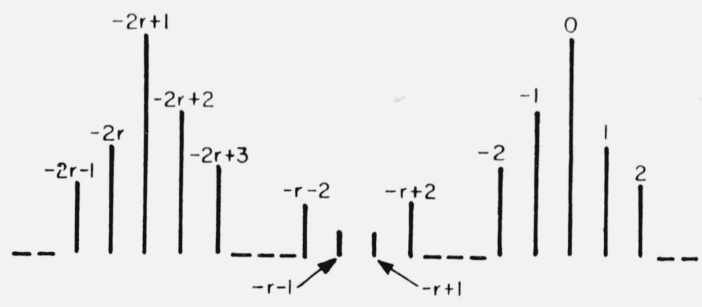

(a) $m$ ODD $(m=2 r-1)$

FIGURE 2. The magnitudes of the harmonic coefficients $\mathrm{c}_{\mathrm{n}}(i \nu)$ in the unstable regions.

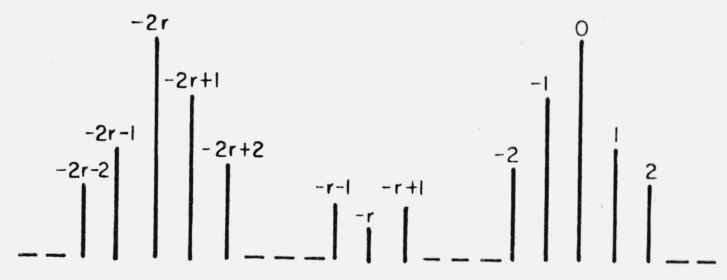

(b) $m$ EVEN $(m=2 r)$ 
To conclude the discussion, it is pertinent to find the magnitudes of the harmonics for small $h^{2}$. In that case, one may approximate $K_{i}$ in the unstable regions as

$$
\frac{1}{K_{i}} \cong L_{i} \cong(m+\Delta+2 i)^{2}-(m+\Delta)^{2} \cong 4 i(m+i) \cong \frac{1}{\bar{K}_{i}} .
$$

For $K_{-i}$, however, condition (4.5) precludes an easy approximate evaluation. The order of magnitude may nevertheless be estimated by replacing $i$ with $-i$ in eq (7.16). One then finds:

$$
\left|\frac{c_{n}}{c_{0}}\right|=\left\{\begin{array}{lc}
\left(\frac{h^{2}}{4}\right)^{n} \frac{m !}{n !(m+n) !}+\mathrm{O}\left(h^{2 n+2}\right) & (n>0) \\
\left(\frac{h^{2}}{4}\right)^{-n} \mathrm{O}\left[\frac{(m+n-1) !}{(-n) !(m-1) !}\right] & (-r<n<0) \\
\left(\frac{h^{2}}{4}\right)^{m+n} \mathrm{O}\left[\frac{(-n-1) !}{(m+n) !(m-1) !}\right] & (-m<n \leqslant-r) \\
\left(\frac{h^{2}}{4}\right)^{-(m+n)} \cdot \frac{m !}{(-m-n) !(-n) !} & \mathrm{O}\left(h^{2-m-n)} \quad(n \leqslant-m)\right.
\end{array}\right.
$$

for the unstable regions. Numerical and approximate analytical considerations indicate that, for $m>3$, the order of approximation for $-m<n<0$ in eqs (7.17) approaches the actual value very closely.

In the stable regions, property (7.10) does not hold any longer and $\bar{K}_{-i}$ turns out to be quantitatively dissimilar from $K_{-i}$. To find the latter, one notes that eqs (3.1) and (3.13) imply that

$$
S-C_{-i} h^{4}=L_{0}-K_{1} h^{4}-C_{-i} h^{4}=\left(K^{-}-C_{-i}\right) h^{4}
$$

where $K^{-}$and $K^{+}=K_{1}$ were defined in eq (3.3). Employing the definition of $C_{-i}$ in eq (3.14) and the series expansion (3.6) for $K^{-}$, one finds

$$
K^{-}-C_{-i}=B_{-i} B_{-(i+1)} h^{4 i}+\mathrm{O}\left[h^{4(i+1)}\right] .
$$

Introducing eqs (7.18) and (7.19) into eq (3.12), one obtains via eqs (3.7) and (3.8)

$$
K_{-i}=A_{-i}+\frac{1}{A_{-(i+1)} h^{4}}+\mathrm{O}\left(h^{0}\right)=\frac{L_{-(i+1)}}{h^{4}}+\mathrm{O}\left(h^{0}\right)=\frac{4(i+1)(i+1-\nu)}{h^{4}}+\mathrm{O}\left(h^{-2}\right) .
$$

In view of the comments following eq (3.22), $\bar{K}_{-i}$ cannot be obtained directly from eq (7.20) and one needs to use definition (3.12) instead. It is then observed that

$$
\bar{S}=\bar{L}_{0}-\bar{K}_{1} h^{4}=(2 m-\nu)^{2}-\lambda-\bar{K}_{1} h^{4} ;
$$

also, an inspection of eqs (4.1)--(4.4) for $\lambda$ reveals that $\bar{S}$ is of $\mathrm{O}\left(h^{0}\right)$, provided $\nu$ is not too close to $m$ (band-edge). Thus

$$
\bar{K}_{-i}=\bar{A}_{-i}+\mathrm{O}\left(h^{4 i}\right)=\frac{1}{\bar{L}_{-i}}+\mathrm{O}\left(h^{4}\right)=\frac{1}{4(m-i)(m-\nu-i)}+\mathrm{O}\left(h^{2}\right) \quad(i \neq m)
$$

and this approximation is valid for use in eqs (7.9) since $i \neq m$ therein.

The terms $K_{i}$ and $\bar{K}_{i}$ are obtained in a similar form to that of the unstable regions. Thus,

$$
K_{i}=\frac{1}{L_{i}}+\mathrm{O}\left(h^{4}\right)=\frac{1}{4 i(i+\nu)}+\mathrm{O}\left(h^{2}\right),
$$




$$
\bar{K}_{i}=\frac{1}{\bar{L}_{i}}+\mathrm{O}\left(h^{4}\right)=\frac{1}{4(m+i)(m-\nu+i)}+\mathrm{O}\left(h^{2}\right) .
$$

Introducing the relations above into eqs (7.9) one has for the stable regions:

$$
\left.\frac{c_{n}}{c_{0}}=\left(-\frac{h^{2}}{4}\right)^{|n|} \frac{\Gamma(1 \pm \nu)}{|n| ! \Gamma(1+|n| \pm \nu)}+\mathrm{O}\left[h^{2(1+|n|)}\right] \text { (for } n \gtrless 0\right)
$$

where $\Gamma(x)$ is the gamma function of argument $x$.

When comparing the last results with eqs (7.17), one recognizes that the order in $h^{2}$ for $\left|c_{n} / c_{0}\right|$ is the same in both stable and unstable regions for $n \geqslant-r$; for $n<-r$, on the other hand, $\left|c_{n} / c_{0}\right|$ changes from $\mathrm{O}\left(h^{2|m+n|}\right)$ to $\mathrm{O}\left(h^{2|n|}\right)$, i.e., by a factor of $h^{2 m}$. This is due to the fact that pairing of the harmonics occurs in the unstable region only.

FIGURE 3. Variation of the harmonic coefficients $\mathrm{c}_{\mathrm{n}}(i \nu)$ within the stable regions, for $\mathrm{h}^{2}=1$.
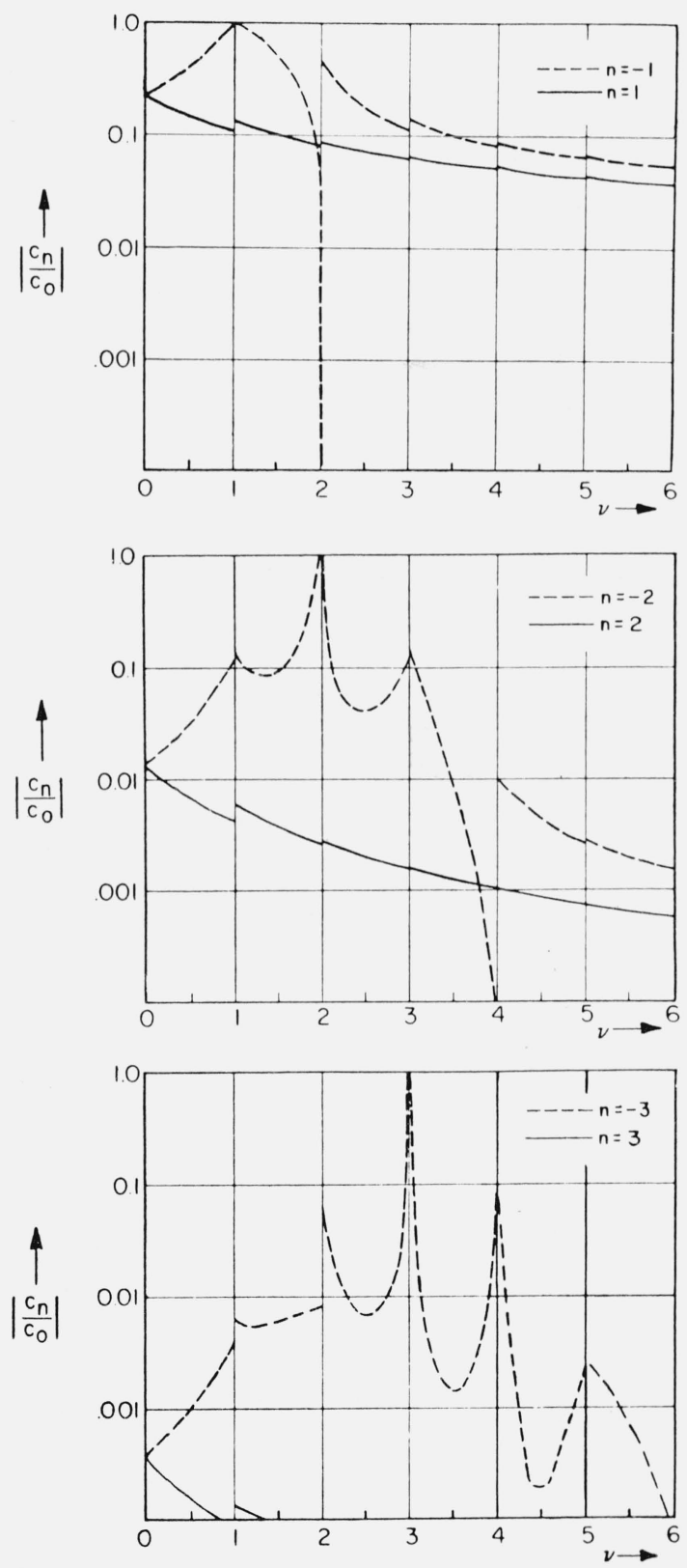
The above considerations indicate the following: (a) for $n \geqslant-r,\left|c_{n}\right|$ decreases monotonically relative to $c_{0}$ as $\lambda$ increases; (b) every harmonic $\left|c_{n}\right|$ with $n<-r$ increases near and at the band edges and decreases towards the middle of the stable bands. These remarks apply to cases with $h^{2}$ small and, as one follows the value of $c_{n}$ with $n<0$, it is clear that this harmonic will behave in the manner (b) up to a value of $\lambda \cong r$ and in the manner (a) for $\lambda>r$. For $n>0$, the harmonic amplitudes behave only in the manner (a) since then $n$ is always greater than $-r$.

The above features are illustrated in figure 3 wherein a few harmonic amplitudes are plotted for the range $0 \leqslant \nu \leqslant 6$ within the stable regions. The vanishing of the $c_{-r}$ harmonic at the left band edge with $m$ even is shown in the figure by $c_{-1}, c_{-2}$, and $c_{-3}$ which vanish respectively, at $\nu=2,4$ and 6. Also, relations (7.16) are verified; thus, $\left|c_{-1}\right|=c_{0}$ at $\nu=1,\left|c_{-2}\right|=c_{0}$ at $\nu=2,\left|c_{1}\right|$ $=\left|c_{-2}\right|$ at $\nu=3$, etc. The constant monotonic behavior of $c_{n}$ for $n>0$ should also be observed.

\section{A Note on Accuracy and Convergence}

In the foregoing derivations, the exact meaning of " $h{ }^{2}$ small" was not explored and this question is examined in the present section.

It is first remarked that, if a numerical computation is desired, the results (3.28) and (3.32) may be valid for the calculation of $\lambda$ for any values of $\nu$ and $h^{2}$. One notes that all the expressions involved are in the form of finite continued fractions, except for $K_{1}=K^{+}$which is infinite. The latter, however, is expandable via eq (3.6) into a series consisting of terms $\kappa_{k}$ that, for large $k$, vary as

$$
\kappa_{k}=B_{k} B_{k+1} h^{4 k} \rightarrow \frac{h^{4 k}}{L_{k+1} \prod_{j=1}^{k} L_{j}} \rightarrow \frac{1}{4(k+1)(m+k+1)}\left[\frac{m !}{k !(m+k) !}\right]^{2}\left(\frac{h}{2}\right)^{4 k}
$$

which converges very rapidly. Hence, for numerical computation, $\lambda$ is obtainable within any desired accuracy provided the iteration process itself converges. However, the convergence of the iteration process is still an open question.

With the above observations in mind, the accuracy of the results for small $h^{2}$ can be examined. As already noted, only the leading terms in the expansions for $K_{1}, A_{1-r}$, etc., need be retained for results up to and including a term of $\mathrm{O}\left(h^{4}\right)$. With respect to $K_{1}$, this means that one needs

$$
h^{4} \ll\left|L_{i} L_{i+1}\right| \text { for all } i>0,
$$

in order to justify the assumed approximation. All of the other functions $A_{1-r}, B_{1-r}$, and $C_{1-r}$ are essentially expressible in terms of $A_{1-r}$; retaining only the first term in the latter means that

$$
h^{4} \ll\left|L_{-j} L_{1-j}\right| \text { for all } 1<j<r
$$

If one replaces $\lambda$ by the first approximation $\lambda_{0}=\nu^{2} \cong m^{2}$ in the two inequalities, one gets:

$$
h^{4} \ll 16 i(i+1)(m+i)(m+i+1) \quad(i>0)
$$

and

$$
h^{4} \ll 16 j(j-1)(m-j)(m-j+1) \quad(1<j<r) .
$$

The worst case for inequality (8.4) is $m=0, i=0$, i.e.,

$$
h^{4} \ll 64
$$

whereas, for (8.5), the worst restriction occurs at $m=5$ and $j=2$ since only $1<j<r$ applies; hence,

$$
h^{4} \ll 12 \times 32 \text {. }
$$




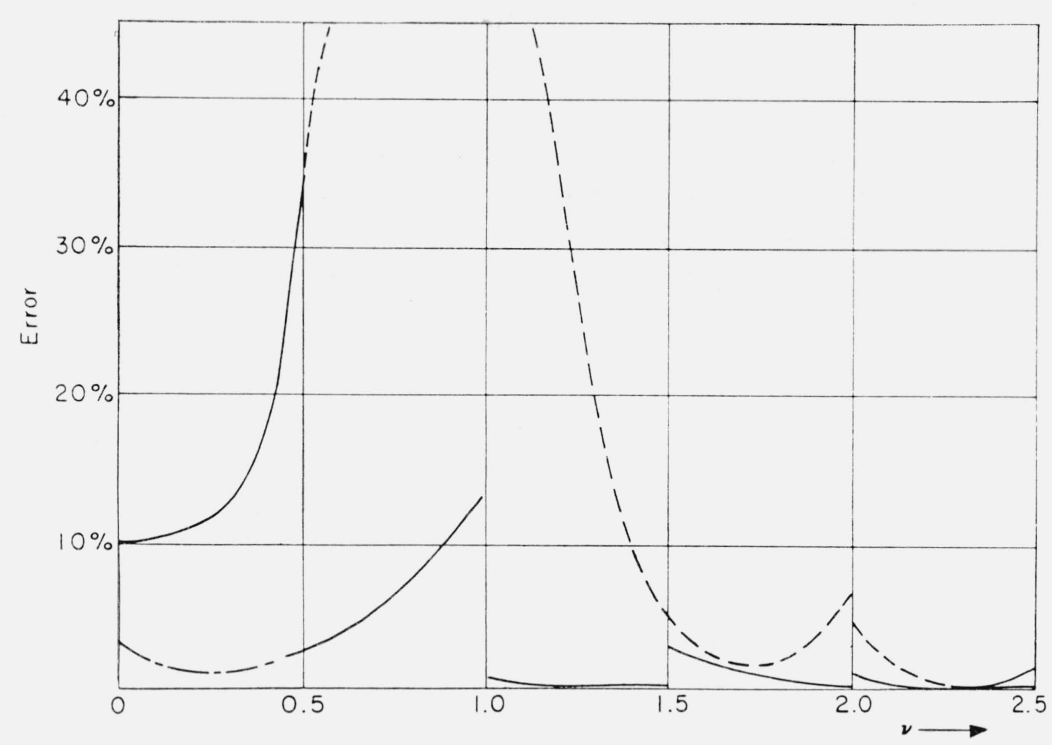

FiguRe 4. Percentage error incurred in the value of $\lambda$, for $\mathrm{h}^{2}=1$.

Solid lines refer to the error produced by eqs (4.1)-(4.4) and dashed lines refer to the error due to the use of eq (1.3). The dot-dashed curve relates to the error which occurs when eq (4.2) is used instead of eq (4.1).

Equations (8.6) and (8.7) indicate that the results obtained should hold well up to and including values of $h^{2}$ of order unity. An analogous examination of $\bar{L}$ (instead of $L$ ) leads to the same restriction. To check these considerations, the exact results for $h^{2}=1$ are compared in figure 4 with those obtained via eqs (4.1)-(4.4). The solid lines indicate the error produced by using eqs (4.1)(4.4) whereas the dashed lines refer to the results obtained via eq (1.3). It is noted that considerable improvement is obtained, especially near $\nu=1$ and $\nu=2$. It is also interesting to observe that smaller errors are obtained if eq (4.2) is used to calculate $\lambda$ for $0 \leqslant \nu \leqslant 0.5$ instead of eq (4.1); this feature is shown in figure 4 by the dot-dashed line. Calculations were also carried out for $h^{2}=0.1$ and 2.5; the errors incurred then are respectively, less than 0.1 percent and 20 percent for all $\nu$, provided eqs (4.2)-(4.4) are employed (i.e., eq (4.2) is extended to cover the range $0<\nu<3 / 2$ ).

The above comments indicate that the formulation discussed in the present paper is useful from both analytical and computational aspects. While it is recognized that the iteration process may not always be convergent, it is also probable that the present formulation might provide a tool for examining the range of validity for some of the expressions of the Mathieu functions and solutions whose convergence is still an open question.

The authors are grateful to Professor A. A. Oliner for his encouragement and Professors E. S. Cassedy and H. Hochstadt for helpful suggestions; also, thanks are due to Dr. Gertrude Blanch, Wright-Patterson Air Force Base, Ohio for her criticism of the manuscript. This work was sponsored by the Air Force Cambridge Research Laboratory, Office of Aerospace Research, under contract No. AF-19 (604)-7499.

\section{Appendix}

Assume that $K_{n}$, as defined in eq (3.11), may be written as

$$
K_{n}=P_{n}+\frac{Q_{n}}{S-R_{n}} \quad(n \leqslant 0)
$$


where $P_{n}, Q_{n}$, and $R_{n}$ are functions to be determined in such a manner that the above becomes an identity; $S$ was defined in eq (3.13). One then has

$$
K_{n-1}=\frac{1}{L_{n-1}-h^{4} K_{n}} \equiv P_{n-1}+\frac{Q_{n-1}}{S-R_{n-1}} .
$$

Substituting for $K_{n}$, eq (A2) yields after some algebraic manipulation,

$$
P_{n-1}+\frac{Q_{n-1}}{S-R_{n-1}} \equiv \frac{1}{L_{n-1}-h^{4} P_{n}}\left[1+\frac{\frac{Q_{n}}{L_{n-1}-h^{4} P_{n}}}{S-R_{n}-\frac{Q_{n}}{L_{n-1}-h^{4} P_{n}}}\right]
$$

and, identifying terms, one obtains

$$
\begin{aligned}
P_{n-1} & =\frac{1}{L_{n-1}-h^{4} P_{n}} \\
Q_{n-1} & =\frac{Q_{n} h^{4}}{\left(L_{n-1}-h^{4} P_{n}\right)^{2}} \\
R_{n-1} & =R_{n}+\frac{Q_{n} h^{4}}{L_{n}-h^{4} P_{n}} .
\end{aligned}
$$

The last relations form a system of difference equations which are solved by noting that the boundary condition at $n=0$ stipulates that

$$
P_{0}=R_{0}=0 \text { and } Q_{0}=1
$$

Applying this to (A4)-(A6), one obtains easily

$$
\begin{gathered}
P_{n}=\frac{1}{L_{n}}-\frac{h^{4}}{L_{n+1}}-\frac{h^{4}}{\mid L_{n+2}}-\ldots-\frac{\left.h^{4}\right\rfloor}{\mid L_{-2}}-\frac{h^{4}}{\sqrt{L_{-1}}} \\
Q_{n}=h^{-4 n} \prod_{i=1}^{-n} P_{-i}^{2} \\
R_{n}=h^{4} \sum_{j=1}^{-n} P_{-j} Q_{1-j}
\end{gathered}
$$

all of which hold for $n<0$ and it is understood that $P_{-1}=1 / L_{-1}$. Upon identifying

$$
A_{n} \equiv P_{n}, \quad B_{n}^{2} \equiv Q_{n} h^{4 n} \text { and } C_{n}=R_{n} h^{-4},
$$

it is readily verified that eq (3.12) is proven and that eqs (3.7), (3.8), and (3.14) are satisfied.

\section{References}

[1] E. Mathieu, J. de Math. Pures \& Appl. (Liouville) 13, 137 (1868).

[2] G. W. Hill, Acta Math. 8, 1 (1886).

[3] N. W. McLachlan, Theory and Application of Mathieu Functions (Clarendon Press, Oxford, 1947).

[4] J. Meixner and F. W. Schäfke, Mathieusche Funktionen und Sphäroidfunktionen (Springer-Verlag, Berlin, 1954).

[5] R. Campbell, Theorie Generale de l'Equation de Mathieu (Masson and Co., Paris, 1955).

[6] National Bureau of Standards, Handbook of Mathematical Functions (U.S. Dept. of Commerce, 1964), ch. 20, p. 721, by Gertrude Blanch.

[7] National Bureau of Standards, Tables Relating to Mathieu Functions (Columbia University Press, N.Y., 1951). 
[8] Lord Rayleigh, Phil. Mag. 24, 145 (1887).

[9] L. Brillouin, Wave Propagation in Periodic Structures, ch. 8, p. 172 (Dover Publications, Inc., N.Y., 1953).

[10] T. Tamir, H. C. Wang and A. A. Oliner, IEEE Trans. on Microwave Theory and Tech. MTT-12, 323 (1964).

[11] N. Minorsky, Nonlinear Oscillators, ch. 16.4 and 20. (Van Nostrand Co., Inc., Princeton, N.J., 1962).

[12] T. Tamir, Math. Comput. 16, 100 (1962).

[13] J. Meixner and F. Schäfke, op. cit., ch. 2.25, p. 119.

[14] F. W. Schäfke, R. Ebert and H. Groh, Num. Math., 4, 1 (1962).

[15] B. van der Pol and M. J. O. Strutt, Phil. Mag. 5, 18 (1928).

[16] O. Perron, Die Lehre von Kettenbrüchen, ch. II-45, p. 205. (Chelsea Publishing Co., N.Y., 1950).

[17] M. Bell, Proc. Glasgow Math. Assoc. 3, 132 (1957).

[18] D. M. Levy and J. B. Keller, Comm. Pure and Appl. Math. 16, 469 (1963).

[19] H. Hochstadt, Comm. Pure and Appl. Math. 17, 251 (1964). 